\title{
Multivariate analysis of factors for failed continuous bladder irrigation in hemorrhagic cystitis patients after hematopoietic stem cell transplantation
}

Wenbo Yang ${ }^{\dagger}$, Yiqing Du ${ }^{\dagger}$, Zhan Qu, Wenjun Bai, Luping Yu, Xiaopeng Zhang, Qi Wang, Xiaowei Zhang, Qing Li and Tao $X u^{*}(D)$

\begin{abstract}
Background: Continuous bladder irrigation (CBI) and proper adjustment of saline irrigation speed are important to avoid CBI failure in hemorrhagic cystitis (HC) patients after allogeneic hematopoietic stem cell transplantation (HSCT). Nevertheless, too fast irrigation speed could take away the patient's much heat, contribute to blood coagulopathy, and increase the nursing workload. Evaluation of risk for $\mathrm{CBI}$ failure remains an unmet clinical need.

Methods: The general information, clinical characteristics, and consultation records of HC patients in 1380 patients with hematopoietic stem cell transplantation in our center from 2017 to 2019 were analyzed retrospectively. The receiver operating characteristic (ROC) curve was used to calculate the cutoff point of the continuous variable, and multivariate logistic regression was used to analyze the risk factors affecting CBI failure in $\mathrm{HC}$ patients.

Results: The incidence of HC after HSCT was 23\%. A total of 227 patients with HC above grade 2 were included. Univariate analysis showed that CRP, age, platelet counts, onset time after transplantation, albumin, and hemoglobin were associated with $\mathrm{CBI}$ failure in the short-term $(P<0.05)$. ROC curve and multivariate logistic regression analysis showed that $C R P>8.89 \mathrm{ng} / \mathrm{ml}(\mathrm{RR}=7.828,95 \% \mathrm{Cl} 2.885-21.244)$, age $<14.5$ years ( $\mathrm{RR}=9.940,95 \% \mathrm{Cl} 3.219-30.697)$, and onset time of $\mathrm{HC}>37 \mathrm{~d}$ after transplantation ( $\mathrm{RR}=7.021,95 \% \mathrm{Cl} 2.204-22.364)$, were independent risk factors for failure of $\mathrm{CBI}(P<0.05)$.

Conclusions: The study identified CRP $>8.89 \mathrm{ng} / \mathrm{ml}$, age $<14.5$ years, and onset time of $\mathrm{HC}$ after HSCT $>37 \mathrm{~d}$ are independent factors for failure of CBI, which could be combined to allow stratification of HC after HSCT patients into low-, intermediate- and high-risk subgroups of CBI failure.
\end{abstract}

Keywords: Continuous saline bladder irrigation, Transplant-related adverse events, Hemorrhagic cystitis, C-reactive protein, Hematopoietic stem cell transplantation, CRP

*Correspondence: xutao@pkuph.edu.cn

tWenbo Yang and Yiqing Du have contributed equally to this work Department of Urology, Peking University People's Hospital, 11 South Xizhimen Street, Beijing 100044, China

\section{Background}

Hemorrhagic cystitis (HC) after allogeneic hematopoietic stem cell transplantation (HSCT) is characterized by diffuse inflammation and hemorrhage of the bladder mucosa. Its clinical manifestation, severity, and prognosis vary greatly. It has been reported that the incidence of $\mathrm{HC}$, as one of the major complications in allogeneic 
HSCT, is $14-30 \%$ [1-4]. Refer to the Droller's HC classification (Table 1 ), grade I means only microscopic haematuria, and gross hematuria means grade II or higher. Conservative observation, hydration, alkalization of urine, diuretics, and antiviral therapy were efficient for most $\mathrm{HC}$ patients with grade I or II, while continuous bladder irrigation (CBI) was required for some grade II, III, and IV patients to avoid urinary tract obstruction caused by blood clots in the bladder.

Patients with this allogeneic HSCT have abnormal immunity, coagulopathy [5], and graft-versus-host disease (GVHD). For urinary tract obstruction of HSCT patients, surgical treatment is associated with mortality and effects were minimal. CBI and proper adjustment of saline irrigation speed are important to avoid CBI failure. Bladder irrigation pressure and speed were dependent on the severity of hematuresis, risk of clots and patient's comfort. As the irrigation continues, the urine should become pink and clear, especially in severe hematuresis [6]. However, we should maintain patient's comfort at the same time. Nevertheless, too fast irrigation speed could take away the patient's much heat, contribute to blood coagulopathy, and increase the nursing workload.

Doctors often believe that the grading of $\mathrm{HC}$ is the most reliable factor to predict the failure of $\mathrm{CBI}$ for $\mathrm{HC}$, which is not practical in clinical practice. Many patients were diagnosed with grade IV of $\mathrm{HC}$ as bladder irrigation failed. It means $\mathrm{HC}$ grade classification of the moment is not of enough practical significance in guiding the saline irrigation speed for $\mathrm{HC}$ to prevent CBI failure. Therefore, it is an unsolved clinical need to predict reliably the CBI failure of $\mathrm{HC}$. We hypothesized that patients with $\mathrm{HC}$ grade II or higher had some clinical characteristics to predict the risk of CBI failure. We report an analysis of $\mathrm{HC}$ incidence and treatment outcome in our center.

\section{Methods}

The study was approved by the institutional review board in our center. The general information, clinical characteristics, and consultation records of HC patients in 1380 patients with HSCT in our center from 2017 to 2019 were analyzed retrospectively. According to the consultation records in our center, if the irrigation line was completely blocked in CBI, it is determined as CBI failure of

\section{Table 1 Hemorrhagic cystitis grade}

\begin{tabular}{ll}
\hline Grade I & Microscopic hematuria \\
\hline Grade II & Macroscopic hematuria \\
Grade III & Macroscopic hematuria with small clots \\
Grade IV & $\begin{array}{c}\text { Gross hematuria with massive clotting, causing urinary } \\
\text { tract obstruction, requiring instrumentation for clot } \\
\text { evacuation }\end{array}$ \\
\hline
\end{tabular}

the patient in our center from 2017 to 2019. The following clinical parameters were collected: general information, the primary diseases, onset time of $\mathrm{HC}$ after HSCT, haploidentical HSCT, sex-mismatch in recipients, cytomegalovirus (CMV) viremia, EB viremia, hemoglobin, platelets, serum creatinine, C-reactive protein (CRP), and serum albumin in the presence of gross hematuria. If gross hematuria or clots are persistent, CBI is indicated in our institute from 2017 to 2019. All the patients with $\mathrm{HC}$ above grade 2 were treated with hydration, alkalization, diuretic equally. When viremia was found, ganciclovir or sodium phosphonate was used for antiviral treatment and human immunoglobulin to enhance immunity treatment until the viremia turned negative.

SPSS 26.0 statistical software was used for data analysis and data processing. The receiver operating characteristic (ROC) curve was used in measurement data to determine the appropriate cut-off point of predicting CBI failure, by which measurement variables were divided into binary variables. Univariate analysis and multivariate logistic regression were used to analyze the risk factors affecting $\mathrm{CBI}$ failure in $\mathrm{HC}$ patients. Comparisons of the rate of $\mathrm{CBI}$ failure across groups were made using analysis of chi-squared tests for categorical and binary variables. The difference was statistically significant at 0.05 .

\section{Results}

Among the 1380 patients with allogeneic HSCT in our center, a total of $311(22.5 \%)$ patients were diagnosed with HC. HC was grade I, II, III, and IV in $84(27.0 \%), 107$ (34.4\%), 73 (23.5\%), and 47 patients (15.1\%), respectively. Among these patients, 227 patients with $\mathrm{HC}$ of degree 2 or higher were included. Table 2 shows the demographic and clinical characteristics of these patients. The patients' primary diseases included acute myeloid leukemia (53, $23.3 \%)$, myelodysplastic syndrome $(25,11.0 \%)$, acute lymphoblastic leukemia $(91,40.1 \%)$, acute mixed cell leukemia (6, 2.6\%), Hodgkin's lymphoma (3, 1.3\%), aplastic anemia $(32,14.1 \%)$, T-cell lymphoma $(1,0.4 \%)$, and others $(16,7.0 \%)$.

A total of 93 in 311 (29.9\%) patients with severe hematuria were undergone CBI. Among $311 \mathrm{HC}$ patients, a total of 39 patients (12.5\%) failed in CBI. Among them, 19 cases underwent cystoscopy to remove blood clots and urinary tract obstruction after failed manual flushing by experienced urologists, and 3 cases were transferred to the pediatric centers for cystoscopy due to their age.

\section{Establishment of the cut-off value of the continuous variable}

To determine the cutoff points of CRP, age, platelets count, post-transplantation onset time, serum albumin, and hemoglobin in the entire cohort, the ROC curve 
Table 2 Patients of grade II and above characteristics

\begin{tabular}{ll}
\hline Number of patients & $\mathbf{2 2 7}$ \\
\hline Age (mean \pm SD), years & $27.0 \pm 14.5$ \\
Women, $\mathrm{n}(\%)$ & $104(45.8 \%)$ \\
Grade, $\mathrm{n}(\%)$ & \\
II & $107(47.1 \%)$ \\
III & $73(32.2 \%)$ \\
IV & $47(20.7 \%)$ \\
Primary diseases, $\mathrm{n}(\%)$ & \\
Acute myeloid leukemia & $53(23.3 \%)$ \\
Myelodysplastic syndrome & $25(11.0 \%)$ \\
Acute lymphoblastic leukemia & $91(40.1 \%)$ \\
Acute mixed cell leukemia & $6(2.6 \%)$ \\
Hodgkin's lymphoma & $3(1.3 \%)$ \\
Aplastic anemia & $32(14.1 \%)$ \\
T-cell lymphoma & $1(0.4 \%)$ \\
Others & $16(7.0 \%)$ \\
CMV virus positivity, $\mathrm{n}(\%)$ & $84(37.0 \%)$ \\
EB virus positivity, $\mathrm{n}(\%)$ & $35(16.3 \%)$ \\
Cyclophosphamide exposure, $\mathrm{n}(\%)$ & $201(88.5 \%)$ \\
CBI failure & $39(17.2 \%)$ \\
\hline
\end{tabular}

for CBI failure was plotted (Fig. 1). The area under the curve (AUC) of CRP was 0.784 . The sensitivity and the specificity were 0.769 and 0.755 respectively as a CRP of $8.89 \mathrm{mg} / \mathrm{dL}$ was defined as the cutoff point. As for the onset time of $\mathrm{HC}$ after HSCT, the AUC was 0.773 and the cutoff value was determined as $37 \mathrm{~d}$ by ROC curve, with a specificity of 0.660 and a sensitivity of 0.821 . The cutoff values of age, platelet count, albumin, and hemoglobin were 14.5 years, $29.5 \times 10^{9} / \mathrm{L}, 36.05 \mathrm{~g} / \mathrm{L}$, and $83.5 \mathrm{~g} / \mathrm{L}$, respectively. Then the impact of CRP and other continuous variables were recoded into binary variables and evaluated in the univariate analysis according to the cutoff point.

\section{Univariate analysis of CBI failure (Table 3)}

In this study, among the 123 male patients, 19 (8.4\%) were failed in CBI, compared with 20 among 104 female patients $(8.8 \%)$. There was no correlation between gender and CBI failure $(P=0.451)$. The age in the whole cohort was $27.0 \pm 14.5$ (IQR 15-36) years. Among the 54 patients with age $<14.5$ years, $16(7.0 \%)$ failed in CBI, and among the 173 patients with age $\geq 14.5$ years, $23(70.4 \%)$ failed in CBI. Age $<14.5$ years was significantly associated with $\mathrm{CBI}$ failure $(\mathrm{RR}=2.746,95 \%$ CI $1.322-5.702$, $P=0.005)$.

In 227 patients, the onset time of $\mathrm{HC}$ after HSCT was right skewness, and the median and mean for the whole cohort was available for $33.0 \mathrm{~d}$ and $59.1 \mathrm{~d}$ (IQR 24.0 d-60.0 d). Among the 96 patients with an onset time of $\mathrm{HC}$ after HSCT more than $37 \mathrm{~d}, 32$ (33.3\%) underwent CBI failure. On the contrary, only 7 (5.3\%) among the 131 patients with an onset time of HC after HSCT fewer than 37d failed in CBI. Onset time of HC after HSCT more than $37 d$ was significantly associated with CBI failure in univariate analysis ( $\mathrm{RR}=8.857,95 \%$ CI 3.704-21.177, $P<0.001$ ).

In the study, the mean and median of CRP in the whole cohort were available for $12.08 \mathrm{mg} / \mathrm{dL}$ and $4.08 \mathrm{mg} / \mathrm{dL}$ (IQR 1.21-11.84). Among the 151 patients with $C R P \leq 8.89 \mathrm{mg} / \mathrm{dL}, 9$ (5.96\%) underwent CBI failure. Among the 76 patients with CRP $>8.89 \mathrm{mg} / \mathrm{dL}, 30$ (39.47\%) underwent CBI failure. $\mathrm{CRP}>8.89 \mathrm{mg} / \mathrm{dL}$ was significantly associated with $C B I$ failure $(R R=10.290$, $95 \%$ CI 4.551-23.264, $P<0.005)$. In the univariate analysis palates count $<29.5 \times 10^{9} / \mathrm{L}(\mathrm{RR}=2.724,95 \%$ CI $1.335-$ 5.558, $P=0.005)$, albumin $<36.05 \mathrm{~g} / \mathrm{L}(\mathrm{RR}=3.943,95 \%$
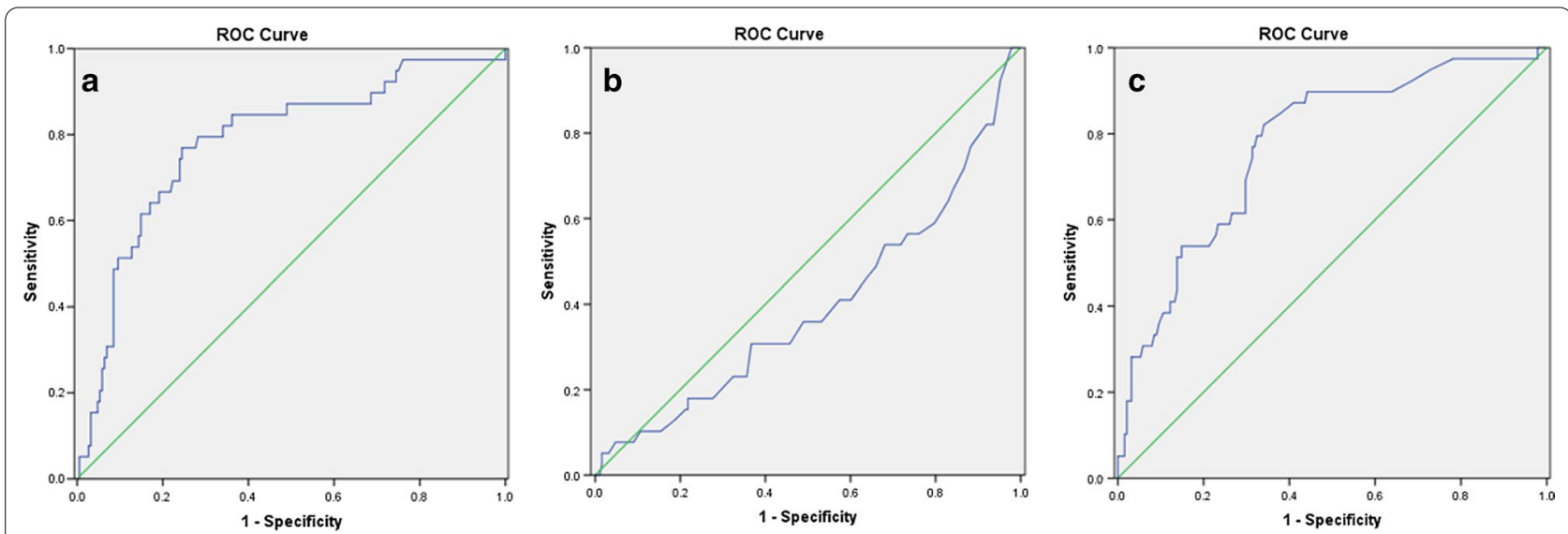

Fig. 1 ROC curve to predict CBI failure. The values plotted on the curves indicate specificity and sensitivity at the points that are closest to the upper-left corner. a CRP, $\mathbf{b}$ age, $\mathbf{c}$ onset time of HC 
Table 3 Results of uni- and multivariate analyses

\begin{tabular}{|c|c|c|c|c|c|c|}
\hline \multirow[t]{2}{*}{ Variable } & \multicolumn{3}{|c|}{ Univariate analyses } & \multicolumn{3}{|c|}{ Multivariate analyses } \\
\hline & $\mathbf{R R}$ & $P$ & $95 \% \mathrm{Cl}$ & $\mathbf{R R}$ & $P$ & $95 \% \mathrm{Cl}$ \\
\hline Age $<14.5$ years & 2.746 & 0.005 & $1.322-5.702$ & 9.940 & 0.000 & 3.219-30.697 \\
\hline Male gender & 0.767 & 0.451 & $0.385-1.531$ & & & \\
\hline Haploidentical HSCT & 0.728 & 0.683 & $0.158-3.362$ & & & \\
\hline Gender difference & 0.965 & 0.926 & $0.454-2.052$ & & & \\
\hline Onset time of $\mathrm{HC}>37 \mathrm{~d}$ & 8.857 & 0.000 & $3.704-21.177$ & 7.021 & 0.001 & $2.204-22.364$ \\
\hline platelet count $<29.5^{*} 10^{9} / \mathrm{L}$ & 2.724 & 0.005 & $1.335-5.558$ & 1.892 & 0.186 & $0.736-4.862$ \\
\hline Albumin $<36.05 \mathrm{~g} / \mathrm{L}$ & 3.943 & 0.004 & $1.473-10.553$ & 2.377 & 0.145 & $0.741-7.623$ \\
\hline $\mathrm{CRP}>8.89 \mathrm{mg} / \mathrm{dL}$ & 10.290 & 0.000 & $4.551-23.264$ & 7.828 & 0.000 & $2.885-21.244$ \\
\hline hemoglobin $<83.5 \mathrm{~g} / \mathrm{L}$ & 3.010 & 0.002 & $1.468-6.172$ & 1.537 & 0.378 & $0.591-4.001$ \\
\hline CMV positivity & 1.078 & 0.836 & $0.530-2.192$ & & & \\
\hline EB positivity & 1.415 & 0.434 & $0.591-3.385$ & & & \\
\hline
\end{tabular}

Gender difference: gender difference between donor and recipient

CI 1.473-10.553, $P=0.004)$, and hemoglobin $>83.5 \mathrm{~g} / \mathrm{L}$ $(\mathrm{RR}=3.010,95 \% \mathrm{CI} 1.468-6.172, P=0.002)$ were also significantly associated with CBI failure.

Nevertheless, in the univariate analysis gender difference between donor and recipient, primary disease type, haploidentical HSCT, CMV positivity, and EB positivity $(P>0.05)$.

\section{Multivariate analysis of CBI failure (Table 3)}

In multivariate analysis, $C R P>8.89 \mathrm{ng} / \mathrm{ml}(\mathrm{RR}=7.828$, 95\% CI $2.885-21.244, \quad P<0.001), \quad$ age $<14.5$ years ( $\mathrm{RR}=9.940,95 \%$ CI 3.219-30.697, $P<0.001)$, and onset time of $\mathrm{HC}>37 \mathrm{~d}$ after transplantation $(\mathrm{RR}=7.021,95 \%$ CI 2.204-22.364, $P=0.001)$ were independent risk factors for failure of $\mathrm{CBI}$.

\section{Risk groups of CBI failure}

Each patient was then assigned to one of three risk groups: those with zero or one risk factors (favorable risk), those with two risk factors (intermediate risk), and those with three risk factors (poor risk). Of the 160 (42\%) patients deemed to be in the favorable-risk group, only 8 (5\%) failed in CBI. In contrast, $88.9 \%$ of the poor-risk group, failed in CBI. There was a significant difference in the rate of $\mathrm{CBI}$ of the three risk groups $(P<0.0001)$.

\section{Discussion}

Urologists are often consulted by hematologists about the failure of $\mathrm{CBI}$ in $\mathrm{HC}$ patients, which may require manual processing of blood clots and even surgical intervention in patients with immunity deficiency and hematopoiesis. In grade II, III, and all IV of $\mathrm{HC}$ patients related to HSCT, CBI is suggested to prevent large blood clotting in bladder forming and lower urinary tract obstruction to allow adequate catheter drainage. Although adjusting the speed of bladder saline irrigation dependent on the color of the drainage from the catheter can avoid the failure of CBI to some extent [7], medical nursing providers always slow down the speed of irrigation in any grades of $\mathrm{HC}$ patients for various reasons limiting CBI prophylactic value, such as hypothermia $[4,8]$. Notably, if CBI failure was not resolved in time and CBI was not suspended, iatrogenic rupture of the bladder or renal function damage could be caused. Evaluation of risk for CBI failure remains an unmet clinical need. Compared with radiation cystitis, although the pathological changes and symptoms of $\mathrm{HC}$ related to $\mathrm{HSCT}$ are mainly in the bladder, its diagnosis and treatment can not bypass the special immune deficiency and abnormal hematopoietic function of HSCT, which means higher significant morbidity and mortality in surgical treatment. Through a large sample retrospective study in our center, the data of 1380 patients who received HSCT were analyzed retrospectively. The incidence of $\mathrm{HC}$ after HSCT was $23 \%$ in our center. The CRP, platelet count, and hemoglobin of patients with gross hematuria were analyzed statistically, and the independent risk factors of CBI failure were discussed. In the study, CRP was firstly found to be an independent risk factor for CBI failure, and its cutoff point $\mathrm{CRP}>8.89 \mathrm{mg} / \mathrm{dl}$ was determined. Up to the date, CRP has not been reported as independent risk factors for $\mathrm{HC}$ grade or treatment outcome. In the current study, we have confirmed that patients with the onset time $>37 \mathrm{~d}$ of $\mathrm{HC}$ after transplantation are more likely to fail in CBI. This is consistent with previous studies. Johnston et al. found that delayed $\mathrm{HC}$ onset time was an independent risk factor for $\mathrm{CBI}$ of $\mathrm{HC}$ in 
children [1]. Our study further determined the cutoff point and confirmed and that $\mathrm{HC}$ onset time $>37 \mathrm{~d}$ is still an independent risk factor for CBI failure.

Within 3 days after the pretreatment of allogeneic HSCT, HC was mainly contributed to the toxicity of cyclophosphamide $(\mathrm{CY})$ in the pretreatment. When acrolein, the metabolite of $\mathrm{CY}$, combines with the epithelial tissue of bladder mucosa, it will cause extensive damage to the mucosa of the patients. At the same time, acrolein is more likely to form crystals and deposits in the kidney or bladder in the acid environment, which aggravates the bleeding and necrosis of the mucosa. Nevertheless, previous studies showed that the $\mathrm{HC}$ after 3 days of HSCT was mainly related to the infection of CMV, adenovirus, BKV [4], JC virus [9], and so on. Stanchi [10] et al. reviewed adverse events of 214 recipients undergoing HSCT in children, and the results indicated that CRP increased significantly during sepsis / SIRS, bacteremia graft rejection, liver or intestinal GVHD, and viremia. In this study, CRP in hematuria was found to be an independent risk factor for CBI failure in HC after HSCT, and CRP $>8.89 \mathrm{mg} /$ $\mathrm{dl}$ of gross hematuria stage is superior to single virus detection.

The urethra is more narrow in younger patients, which is closely associated with the CBI failure of HC. Lucila et al. [11]. reviewed 133 recipients undergoing HSCT and found that younger patients were more likely to develop BK polyomavirus related $\mathrm{HC}$ in multivariate regression analysis and had a worse prognosis. Nevertheless, in Johnston,s study, age as a continuous variable was not associated with $\mathrm{HC}$ treatment outcome $(P=0.0773)$ and HC grade $(P=0.0721)$ [1]. In this study, multiple regression analysis was carried out in the multi-age group of HSCT. It was found that the age of fewer than 14.5 years old was an independent risk factor for the failure of $\mathrm{CBI}$ in $\mathrm{HC}$ patients.

For the applicability of the research results, the study excluded grade I HC patients, for these patients had no gross hematuria or CBI. The limitations of this study are as follows: 1 . This study is retrospective and all data was collected from a single-center. However, our center has received a large number of HSCT patients from all over the country. The source of HSCT patients is rather sufficient. Clinically factors can still be collected from this single-center, which may guide the current treatment and future investigation. 2. The results of CRP, serum albumin, and so on are single time point values, which may vary with time point. But this study chose the initial gross hematuria time stage point as the time point to describe the study for the best fit for the clinical pathway. Despite these limitations, this study revealed the effect of serum $\mathrm{CRP}$ on the failure of CBI in $\mathrm{HC}$ patients.

\section{Conclusions}

$\mathrm{HC}$, as one of the complications of HSCT, is not rare. Compared with radiation cystitis, HSCT related HC has unique clinical characteristics. The study identified CRP $>8.89 \mathrm{ng} / \mathrm{ml}$, age $<14.5$ years, and onset time of $\mathrm{HC}$ after $\mathrm{HSCT}>37 \mathrm{~d}$ are independent factors for failure of $\mathrm{CBI}$, which could be combined to allow stratification of $\mathrm{HC}$ after HSCT patients into low-, intermediate- and high-risk subgroups of CBI failure. In the clinical pathway, we can avoid the dilemma of surgical intervention by taking more targeted and active interventions. In the future, we need to expand the sample size and conduct the prospective, multi-center study to gradually improve the predicted model.

\section{Abbreviations}

CBI: Continuous bladder irrigation; CRP: C-reactive protein; CY: Cyclophosphamide; CMV: Cytomegalovirus; GVHD: Graft-versus-host disease; HSCT: Hematopoietic stem cell transplantation; HC: Hemorrhagic cystitis; ROC: Receiver operating characteristic.

\section{Acknowledgements}

Not applicable.

\section{Authors' contributions}

YWB and XT conceived and designed the study, YWB, DYQ, QZ, BWJ, YLP, ZXP, $Z X W, L Q$ and WQ performed the clinical data collection. XT and YWB wrote the paper. DYQ, BWJ and LQ reviewed and edited the manuscript. All authors read and approved the final manuscript.

\section{Funding}

The study was funded by the General Project of the National Natural Science Foundation of China (No. 81872086).

\section{Availability of data and materials}

The datasets used and/or analysed during the current study are available from the corresponding author on reasonable request.

\section{Ethics approval and consent to participate}

The study was approved by the Institutional Ethics Committee Board of Peking University peoples' hospital. Consent to participate was deemed to be not required.

\section{Consent for publication}

Not applicable.

\section{Competing interests}

The authors declare that they have no competing interests.

Received: 9 July 2020 Accepted: 2 November 2020

Published online: 10 November 2020

\section{References}

1. Johnston D, Schurtz E, Tourville E, Jones T, Boemer A, Giel D. Risk factors associated with severity and outcomes in pediatric patients with hemorrhagic cystitis. J Urol. 2016;195(4 Pt 2):1312-7.

2. Philippe M, Ranchon F, Gilis L, Schwiertz V, Vantard N, Ader F, LabussiereWallet $H$, Thomas $X$, Nicolini FE, Wattel $E$, et al. Cidofovir in the treatment of BK virus-associated hemorrhagic cystitis after allogeneic hematopoietic stem cell transplantation. Biol Blood Marrow Transpl J Am Soc Blood Marrow Transpl. 2016;22(4):723-30. 
3. Wu Q, Zhou F, Song NX, Liu XM, Yu Z, Song XC, Li X, Zhang H. Clinical features and risk factors of hemorrhagic cystitis after allogeneic hematopoietic stem cell transplantation. Zhonghua xue ye xue za zhi = Zhonghua xueyexue zazhi. 2019;40(3):187-90.

4. Cesaro S, Dalianis T, Hanssen Rinaldo C, Koskenvuo M, Pegoraro A, Einsele $\mathrm{H}$, Cordonnier C, Hirsch HH. ECIL guidelines for the prevention, diagnosis and treatment of BK polyomavirus-associated haemorrhagic cystitis in haematopoietic stem cell transplant recipients. J Antimicrob Chemother. 2018;73(1):12-21.

5. Cheuk DK, Chiang AK, Ha SY, Chan GC. Interventions for prophylaxis of hepatic veno-occlusive disease in people undergoing haematopoietic stem cell transplantation. Cochrane Database Syst Rev. 2015;5:Cd009311.

6. Scholtes S. Management of clot retention following urological surgery. Nurs Times. 2002;98(28):48-50

7. Moslemi MK, Rajaei M. An improved delivery system for bladder irrigation. Ther Clin Risk Manag. 2010;6:459-62.

8. Cao J, Sheng X, Ding Y, Zhang L, Lu X. Effect of warm bladder irrigation fluid for benign prostatic hyperplasia patients on perioperative hypothermia, blood loss and shiver: a meta-analysis. Asian J Urol. 2019;6(2):183-91.

9. Tzannou I, Papadopoulou A, Naik S, Leung K, Martinez CA, Ramos CA, Carrum G, Sasa G, Lulla P, Watanabe A, et al. Off-the-shelf virus-specific T cells to treat BK virus, human herpesvirus 6, cytomegalovirus, epstein-barr virus, and adenovirus infections after allogeneic hematopoietic stem-cell transplantation. J Clin Oncol. 2017;35(31):3547-57.

10. Cabanillas Stanchi KM, Queudeville M, Malaval C, Feucht J, Schlegel P, Dobratz M, Seitz C, Müller I, Lang P, Handgretinger R, et al. Comparison of procalcitonin and C-reactive protein as early diagnostic marker for the identification of transplant-related adverse events after allogeneic hematopoietic stem cell transplantation in pediatric patients. J Cancer Res Clin Oncol. 2019:145(11):2779-91.

11. Kerbauy LN, Kerbauy MN, Bautzer V, Chapchap EC, de Mattos VRP, da Rocha JDA, Esteves I, Kutner JM, Kerbauy FR, Ribeiro AAF, et al. Severe hemorrhagic cystitis caused by the BK polyomavirus is associated with decreased survival post-allogeneic hematopoietic stem cell transplantation. Transpl Infect Dis. 2019;21(5):e13101.

\section{Publisher's Note}

Springer Nature remains neutral with regard to jurisdictional claims in published maps and institutional affiliations.
Ready to submit your research? Choose BMC and benefit from:

- fast, convenient online submission

- thorough peer review by experienced researchers in your field

- rapid publication on acceptance

- support for research data, including large and complex data types

- gold Open Access which fosters wider collaboration and increased citations

- maximum visibility for your research: over $100 \mathrm{M}$ website views per year

At BMC, research is always in progress.

Learn more biomedcentral.com/submissions 